\title{
Environment perception simulation for radar stimulation in automated driving function testing
}

\author{
F. M. Maier@, V. P. Makkapati, M. Horn
}

\begin{abstract}
Automated driving is seen as one of the key technologies that shape our future mobility. Testing these automated driving functions (ADF) in virtual environments has the potential to speed up their development and homologation. As the automated driving functions rely on sensors to perceive the environment, a key requirement for virtual testing is the ability to simulate the environment perception of the involved sensors. In this paper we present a concept for environment perception simulation of radar sensors (EPSR) -namely radar signature and stimulation input generation (RASIG) - to be employed in the context of vehicle-in-the-loop (ViL) tests in conjunction with over-the-air (OTA) stimulation hardware. The requirements on environment perception simulation of radar sensors for integration into such a test set-up and its real-time capability along with some validation results are discussed.
\end{abstract}

Keywords: RCS; testing; automotive radar; automated driving functions; environment perception simulation for radar; Phong

\section{Simulation der Umgebungswahrnehmung von Radar-Sensoren für deren Stimulation in Tests von automatisierten Fahrfunktionen.}

\begin{abstract}
Automatisiertes Fahren wird als eine der Schlüsseltechnologien gesehen, welche unsere zukünftige Mobilität formen werden. Ein Testen dieser automatisierten Fahrfunktionen in virtuellen Umgebungen könnte deren Entwicklung und Homologation beschleunigen. Da automatisierte Fahrfunktionen ihre Umgebung mittels Sensoren wahrnehmen, sind Simulationen der Umgebungswahrnehmung, im folgenden kurz Simulationen genannt, aller involvierter Sensoren eine essentielle Voraussetzung für virtuelles Testen. In diesem Artikel präsentieren wir ein Konzept zur Simulation von Radar Sensoren zum Einsatz in Vehicle-in-the-Loop Tests mit over-the-air Stimulationshardware. Die Anforderungen an Simulationen in einem derartigen Testaufbau werden diskutiert. Wir präsentieren unseren Ansatz für solch eine Simulation, die Radarsignatur- und Stimulationsinputgenerierung kurz RASIG und diskutieren deren Echtzeitfähigkeit sowie Validierungsergebnisse.
\end{abstract}

Schlüsselwörter: RCS; automotive Radars; automatisierte Fahrfunktion; Umgebungswahrnemungssimulation; Phong

\section{Introduction}

The automotive industry is working towards one of its most significant changes in history: driving automation. ADFs take control of a part or the whole driving task in some or all traffic scenarios [1]. Testing these automated driving functions in virtual environments has the potential to speed up their development [2]. As ADF rely on sensors to perceive their environment, a key requirement for virtual testing is environment perception simulation of involved sensors (EPSS) which is currently being researched intensively e.g. in the research projects PEGASUS [3] and ENABLES [4]

Given the versatility of driving conditions, the amount of relevant test scenarios for ADF testing is enormous. In virtual testing various environment conditions can be simulated at low cost, whereas at the end of the development chain, the integrated vehicle and it's ADFs has to pass all verification \& validation $(V \& V)$ tests on test tracks or on public roads. This is time consuming, expensive and not all relevant traffic scenarios can be recreated. Thus, testing in stimulated virtual environments has the potential to speed up ADF development and homologation $[2,5]$.

Such an environment for virtual development and validation of ADF is the AVL Driving Cube ${ }^{\mathrm{TM}}[6]$ shown in Fig. 1. Here the vehicle is placed on a ViL test bench and all environment perceiving sensors are stimulated over the air (OTA) according to virtual test scenarios.
Stimulation describes the act of manipulating an entity such that its state is in accordance with an environment scenario despite physically not being in such a scenario, but for e.g. on a test bench. OTA stimulation is required, in order to leave the vehicle and its sensors physically unchanged. Figure 2 shows the basic components required for OTA stimulation of a sensor. First the environment simulation is performed where the test scenario is reconstructed/simulated virtually (refer also to Fig. 1). Then based on this recreated environment the EPSS computes the stimuli to be generated, in case of passive sensors, or parameters for manipulation, in case of active sensors, that are realized by the stimulation hardware in the next stage. Whilst OTA stimulation systems for passive sensors like cameras have been developed [7], stimulating active sensors OTA, esp. lidar and radar is an on-going research topic $[3,4]$.

In this work we consider OTA stimulation of an automotive radar sensor. Section 2 presents first the properties of- and requirements

Maier, F. Michael, Institute of Automation and Control, Graz University of Technology, Inffeldgasse 21b/l, 8010 Graz, Austria (E-mail: f.m.maier@tugraz.at); Makkapati, Vamsi P., Institute of Automation and Control, Graz University of Technology, Inffeldgasse 21b/l, $8010 \mathrm{Graz}$, Austria; Horn, Martin, Institute of Automation and Control, Graz University of Technology, Inffeldgasse $21 \mathrm{~b} / \mathrm{l}, 8010 \mathrm{Graz}$, Austria 


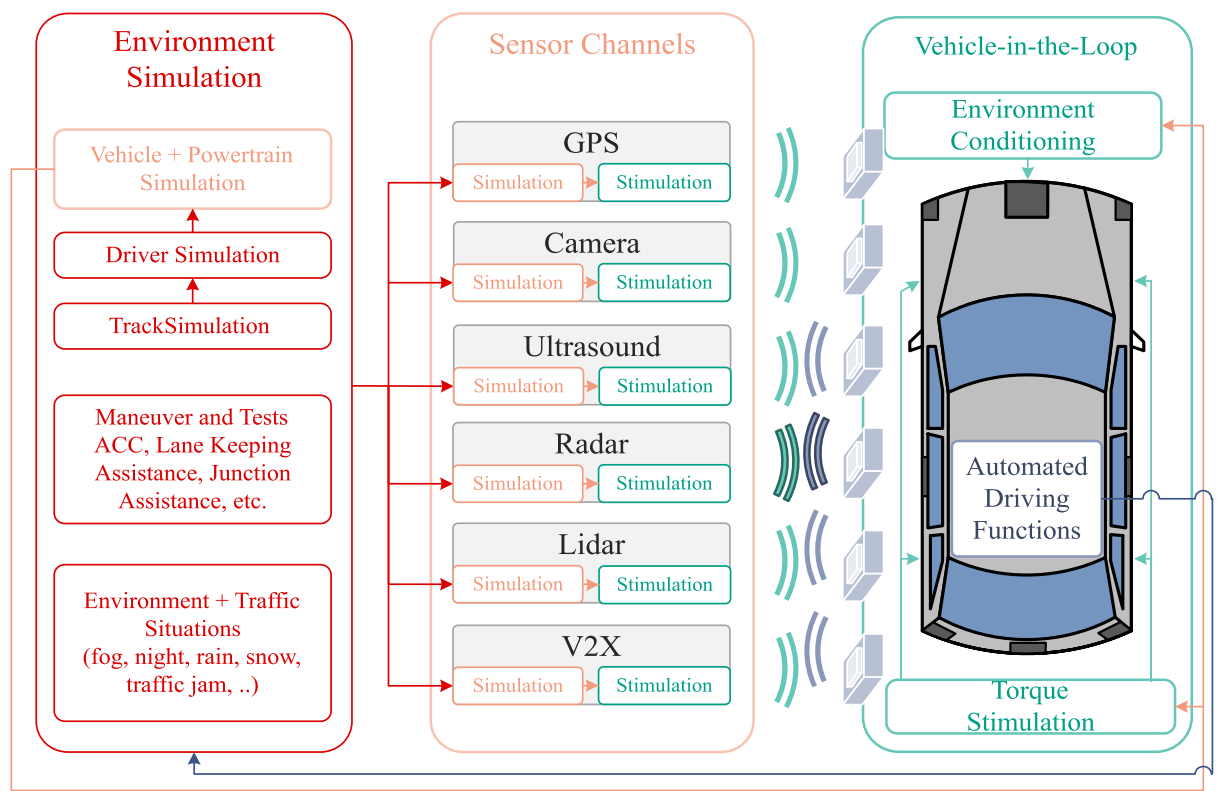

Fig. 1. ViL ADF test system

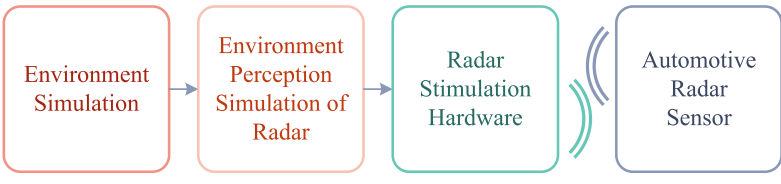

Fig. 2. Components of a radar sensor stimulation chain

for OTA stimulation of radar sensor, and then an overview of existing EPSS for radar sensors. Following this, in Sect. 3 the RASIG concept is presented. Details concerning the implementation of RASIG are discussed in Sect. 4. Before concluding the paper in the last section, validation results of the proposed RASIG method are presented in Sect. 5.

\section{Radar OTA stimulation chain}

The components of the radar stimulation chain as shown in Fig. 2 are discussed, and in doing so requirements for EPSR are derived. For this purpose, first a brief introduction to automotive radar sensors is given. Then the environment simulation with the information available to EPSR is described and radar stimulation units and their required input. Finally requirements on EPSR and a brief state-ofthe-art (SOA) for EPSR are given.

\subsection{Automotive radar sensors}

We consider automotive radar sensors using SoA frequency modulated continuous wave (FMCW) with $77 \mathrm{GHz}$ and 1-4 GHz bandwidth. They send an EM-wave into the field of view (FoV) spanned by azimuth $\phi_{F o V}$ and elevation $\theta_{F o V}$ and measure signals scattered back onto them. This backscattered field is called radar signature (RS). The changes in the RS, compared against the illumination signal from the sensor are quantified in the following quantities (a meaningful range of these quantities is also given [8]):

- Doppler frequency $f_{D}\left( \pm 35 \mathrm{kHz} \Leftarrow v_{\text {rel }}: \pm 252 \mathrm{~km} / \mathrm{h}\right)$,

- distance $r(1 \mathrm{~m}-250 \mathrm{~m} \Rightarrow \Delta t$ : $6.6 \mathrm{~ns}-1.67 \mu \mathrm{s})$,

- azimuth $\phi\left( \pm \phi_{F o V} / 2\right)$,

- power $\operatorname{Pr}\left(-90 \mathrm{dBm}\right.$ to $\left.-10 \mathrm{dBm} \Leftrightarrow \sigma: 1 \mathrm{~m}^{2}-10.000 \mathrm{~m}^{2}\right)$.

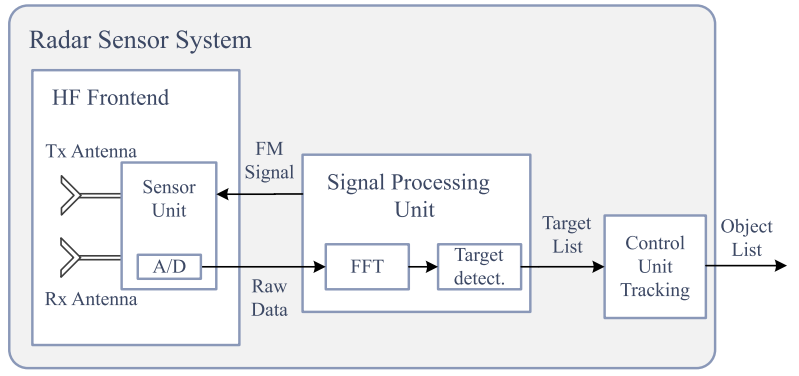

Fig. 3. Overview of radar sensor

The sensing process of automotive radar is shown in Fig. 3, through signal processing (FFT) of the raw data the above mentioned quantities are extracted and then filled into range-azimuth bins. A (rangeazimuth) bin is the smallest unit of quantization in range and azimuth. Each bin contains information in the form of $f_{D}$ and $P_{r}$. Through further data processing and tracking, high-level information in the form object lists (OL) containing position, size and classification of the identified objects is extracted. This high-level data is typically updated every $50 \mathrm{~ms}$ setting the bar for real time constraints on simulation.

\subsection{Environment simulation}

The role of ES is to provide a virtual reality for a test scenario. Available ES software such as $[9,10]$ contains all test scenario objects as 3D triangle meshes and simulates their dynamic behaviour. Each Object is associated with a reference point, which specifies their position in a scenario. During an ADF ViL test any changes in the virtual scenario are communicated to the real vehicle using stimulation chains. Also any control actions taken by the ADF in the vehicle are transferred back into the virtual scenario. This requires, as shown in Fig. 1, a closed loop communication between the entities. Thus computations must be carried out online and in real-time.

In addition to the aforementioned functionality the environment simulation must contain EPSR specific information. Different EPSR 
methods require different kinds of information from the ES. This can be in the form of monostatic radar cross section (RCS) per object, material properties (such as the physical reflection properties, approximated reflection parameters), etc. State of the objects such as position and orientation together with the EPSR information are transmitted to the EPSR

\subsection{Radar OTA stimulation}

A radar OTA stimulation unit manipulates a radar sensor signal such that the perceived radar signature is in accordance with the environment scenario despite physically not being in the scenario, but on a test bench. This requires manipulation of the sensor signal in terms of the quantities mentioned in Sect. 2.1. Since the manipulation is performed on the illumination signal-irrespective of the waveform - the stimulation process is independent of the various modulation techniques used in automotive radars. Current radar stimulation units, are only capable of stimulating point targets [11-14]. Thus the effect of an object on the RS is represented and stimulated through stimulation points (SPS)

$$
\mathrm{SP}=\left[\begin{array}{llll}
f_{D} & \Delta t & \phi & \sigma
\end{array}\right] .
$$

The cumulated power density scattered back from an object is encoded in the RCS value, $\sigma$. Current stimulation hardware is capable of creating up to 4 SPs, most without azimuth information [11, 13, $14]$, and some $[12,15]$ with. Future stimulation units $[16,17]$ will be capable of stimulating areas, rather than points. Thus depending on the stimulation hardware, available, the EPSR should generate appropriate output.

\subsection{Requirements on radar OTA stimulation chain}

The real time step size of the OTA stimulation chain has been chosen at $1 \mathrm{~ms}$ in order to stimulate the radar sensor without affecting its object tracking algorithms. Requirements on the OTA stimulation chain from Sects. 2.1-2.3:

- environment simulation

- EPSR specific information

- OTA stimulation hardware

- EPSR

- signal manipulation parameters encoded into SP

- number of generated SPs $\leq$ number of SPs that the stimulation hardware can handle

- real time $1 \mathrm{~ms}$

- online computation

\subsection{EPSR for OTA stimulation}

EPSR are developed in accordance with the requirements described in Sect. 2.4. They can be distinguished into two parts: Simulation of changes to the illumination signal resulting in RS. And reduction of this RS into SP, which should maximally exploit the capabilities of the employed radar stimulation hardware. While the computation of $f_{D}, \Delta t$ and $\phi$ is straightforward, RCS $\sigma$ computation is an ongoing research topic [18-20] especially under real time constraints as required in EPSR. The methods employed in SOA EPSR for RCS computation can be distinguished as either phenomenological or physical, with increasing degrees of online computation load. Phenomenological models exhibit little to no scenario dependence with regard to received power density. Physical methods compute scenario dependent received power density.

The phenomenological approach is made up of purely object dependent RCS computation methods. Basic models [21, 22] use monostatic RCS measurements of objects and add noise on top.

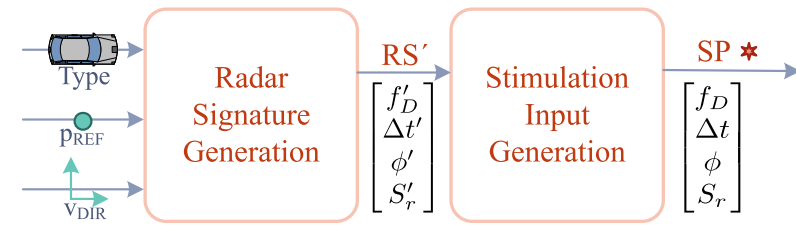

Fig. 4. RASIG components

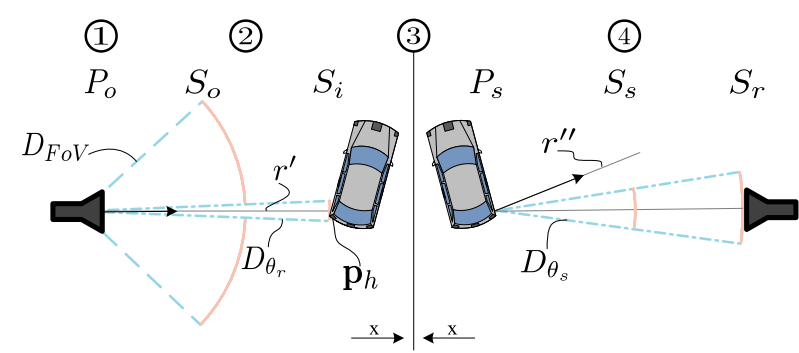

Fig. 5. Single path propagation RS overview

More advanced models represent objects by multiple virtual scattering centers [23]. The advantage of such models is their computational simplicity, as they do not have to compute RCS, but read it from a table. But they can be unrealistic in complex traffic scenarios as they only vary the RCS of an object due to its occlusion, position and orientation with regard to the radar sensor, without considering scenario dependent effects such as partial occlusion and multipath propagation (a usual source of false positives).

The physical approach is made up of scenario dependent RCS computation methods. This includes field simulation software based on first principles (Maxwell's equations) [24]. These methods require multiple hours to solve the EM-field interactions even with simple objects. Less time consuming methods use bidirectional reflectance distribution functions (BRDF) to compute interactions with surfaces. Within RASIG a BRDF method is used.

\section{Radar signature and stimulation input generation (RASIG)} The presented RASIG employs a ray tracing based BRDF approach to RCS computation, for which knowledge of position, orientation, and object data such as material properties of all surfaces in the test scenario are required. Based on this information from the environment simulation RASIG computes SPs in two steps as described in Sect. 2.5 shown in Fig. 4-radar signature generation and stimulation input generation.

\subsection{Radar signature generation}

The radar signature generation computes the changes to the FMCW illumination signal in the form of time delay, azimuth, Doppler and power density. These changes affect the frequency band $76-80 \mathrm{GHz}$ equally. The Doppler frequency shift and the scattering due to interaction with surfaces are frequency dependent, but vary insignificantly across this frequency band. Thus radar signature can be computed for a single frequency without loss of significant information, improving computation time.

To compute the RS, four steps-labelled (1) to (4)-need to be modelled as shown in Fig. 5. These steps are: (1) sending of the illumination signal, (2) its propagation into the scene, (3) its interaction with surfaces and (4) its propagation back to the sensor.

Due to the high frequency $(77 \mathrm{GHz})$ of the illumination signal and the macroscopic nature of test scenarios, far-field conditions 
apply and asymptotic methods can be used. This allows the EM-field as well as its propagation to be quantized into- and approximated by rays, which propagate independent from each other. Each ray ${ }^{1}$ contributes a part RS' to the RS. These rays have associated with them the so-called ray tube with an opening angle of $\phi_{r}$. These ray tubes are so considered that they span the entire FoV, for example using a pyramidal shape. The size of the base surface $A^{\prime}$ of the ray tube depends on ray length $r^{\prime}$ and directionality $D_{\theta}$ of the sent $D_{\theta_{r}}$ or scattered $D_{\theta_{s}}$ wave. For small opening angles $\phi_{r}$ the directionality $D_{\theta}=\phi_{r}^{2}$. Directionality $D_{\theta}$ ranges from $D_{0}$ for a parallel wave to $D_{4 \pi}$ for isotropic radiation, $D_{F o V}$ refers to the FoV of the simulated radar.

$$
A^{\prime}=D_{\theta} r^{\prime 2}
$$

Each ray contains a part of the power sent out $P_{0}$, which is either distributed homogeneously $P_{o}^{\prime}=P_{o} / N_{r}$ with $N_{r}$ being the number of rays, or adjusted according to the antenna radiation pattern. Considering rays allows the calculation of azimuth $\phi^{\prime}$ and time delay $\Delta t^{\prime}$ using the travelled distance $d^{\prime}$, with $d^{\prime}=2 r^{\prime}$ for the first hit. The power $P_{o}^{\prime}$ of a ray is distributed over the cross section of the ray tube as power density $S_{0}^{\prime}$. The interaction with a surface can now be computed at the point where the ray intersects the surface the so-called hit point $\mathbf{p}_{h}$. Additionally, we assume that the intersected surface covers the whole ray tube. This introduces errors especially at the edge of objects. A sufficiently high ray resolution ensures that these errors remain small.

Our approach for modelling the interaction of ray with a surface is based on bidirectional reflection distribution functions (BRDF) [25]. The material properties are characterized by the parameters of BRDF. Within RASIG the so called Phong BRDF [26] which consists of a diffuse reflection and a specular reflection lobe has been adapted to compute the interaction of radar waves with a surface. The adaption has been described in depth in [27] and will hence not be described here. This method can be executed using power density or field strengths, here power density based computations are described and differences between power density and field strength based computations will be discussed in Sect. 3.2. In the simulation at each hit point this BRDF is evaluated using the geometric properties of the interaction, to compute the power density scattered $\left(S_{S}^{\prime}\right)$ back towards the sensor. Then the power density received at the sensor $S_{r}^{\prime}$ after propagating back to sensor is computed. Multipath propagation is equivalent to single path propagation computations except for the following difference. When a ray's intersection with a surface is computed a new reflection ray ${ }^{2}$ is shot in the specular direction with its initial properties being defined by its predecessor. Its power $P_{0}^{\prime \prime}$ is the predecessor power minus the power scattered towards the observer $P_{o}^{\prime \prime}=P_{o}^{\prime}-P_{s}^{\prime}$. The Phong formula is not energy conservative, but with this implementation the ratio of maximum received power to sent power is limited to $\leq 1$. With the ray approximation, hit point, $\Delta t^{\prime}$ and $\phi^{\prime}$ are computed and $S_{S}^{\prime}$ is calculated by evaluating the Phong BRDF. Finally Doppler shift $f_{D}^{\prime}$ is computed according to relative velocity.

Another more precise approach for calculating the scattering of an EM-wave after interaction with a surface using the so-called physical optics (PO) approximation is presented here. It is an intermediate method between geometric optics, which ignores wave effects (like interference, diffraction, polarization), and solving the Maxwell's equations [24]. The basic procedure used to calculate the scattered field at an observation point is as follows

\footnotetext{
${ }^{1}$ Ray quantities are denoted by apostrophe'.

2 Multipath ray quantities are denoted by two apostrophe".
}

- currents induced on the (metallic) surface due to the incident EM field are calculated first,

- then the re-radiated field due to this induced surface current is calculated.

These re-radiated fields can be modelled using Maxwell's equations, which are a set of partial differential equations. One celebrated integral solution (which allows for numerical implementation) to the Maxwell's equations are the so-called Stratton-Chu integrals ${ }^{3}$ [31]. The following approximations allow for simplification of the Stratton-Chu equations and constitute the above mentioned PO method:

- far-field condition

- tangent plane approximation, valid when the curvature of the surface is higher than the wavelength.

As already mentioned at the beginning of this section, the nature of the problem considered in this article satisfies both these conditions. Using these approximations Stratton-Chu equation for the electric field at an observation point is simplified as

$$
\mathbf{E}_{P O}^{S}(\mathbf{r})=\frac{j \omega}{2 \pi} \frac{e^{j k r}}{r} \oiint_{S}\left(\hat{\mathbf{n}} \times \mathbf{B}^{i}\right) e^{-j k \hat{\mathbf{r}} \cdot \mathbf{x}} d a,
$$

where $S$ represents the surface of interest, $\omega$ the frequency of the incident field, $\mathbf{r}=r \hat{\mathbf{r}}$ is the position of the observation point w.r.t. the surface, $\mathbf{B}^{i}$ is the magnetic field, $\hat{\mathbf{n}}$ the normal to the surface, $\mathbf{x}$ is the position of the surface w.r.t. the radar sensor and da is the area of an infinitesimal surface element.

Since the objects within the traffic scenario are represented using triangle meshes, the surface integral in (3) is solved for a triangle numerically. This numerical result is used to compute the contribution of each triangular mesh element in the traffic scenario to the scattered field resulting in the RS. Our implementations have shown that this approach is real time capable for scenarios with small numbers of triangles but unfit for large traffic scenarios with high numbers of triangles. As such this approach shows the limitations of even fast exact high frequency approximations.

\subsection{Stimulation input generation}

To compute SPs, the RS is first split up into separate parts which should then be stimulated together. For single path propagation this separation is achieved by grouping all RS's from an object together. The RS' from multipath propagation cannot be clustered as before, but can be clustered by similar range azimuth and Doppler shift. With this separation, each part of the RS is reduced into a stimulation point. A weighted average using the cumulated power density $S_{r}$ as weight is used for calculating $f_{D}, \Delta t$ and $\phi$ from their corresponding ray equivalents, as follows:

$$
\begin{aligned}
& S_{r}=\sum_{m=0}^{N_{r}} S_{r, m}^{\prime} \\
& d=\frac{1}{S_{r}} \sum_{m=0}^{N_{r}} d_{m}^{\prime} S_{r, m}^{\prime} \\
& f_{D}=\frac{1}{S_{r}} \sum_{m=0}^{N_{r}} f_{D, m}^{\prime} S_{r, m}^{\prime}
\end{aligned}
$$

${ }^{3}$ For the sake of brevity these integral equations are not given here, but interested readers can refer to any book dealing with electromagnetic theory, for example [28-30]. 


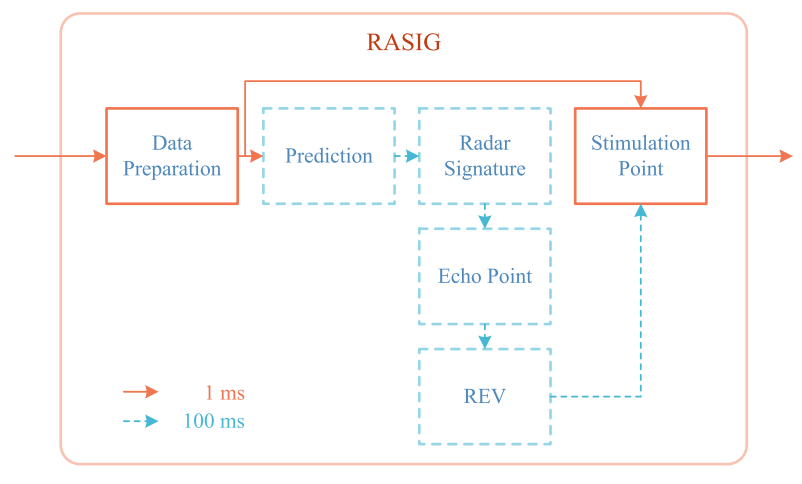

Fig. 6. Implementation RASIG

$$
\begin{aligned}
& \phi=\frac{1}{S_{r}} \sum_{m=0}^{N_{r}} \phi_{m}^{\prime} S_{r, m}^{\prime} \\
& r=\frac{d}{2}, \quad \Delta t=\frac{d}{c},
\end{aligned}
$$

where subscript $m$ is the index of a ray, $d^{\prime}$ the distance travelled by the ray and $c$ is the velocity of light. The RCS is computed using the cumulated power density received $S_{r}$ and the power density incident $S_{i}$ at the averaged distance $r$ of the SP

$$
\begin{aligned}
& S_{i}=\frac{P_{O}}{D_{F o} V r^{2}} \\
& \sigma=4 \pi r^{2} \frac{S_{r}}{S_{i}} .
\end{aligned}
$$

The RCS computations have been described for power densities. The same computations could be done using field strengths as well. Field strength $E_{r}$ computation is phase dependent (12) an thus, sensitive to slight changes in $d^{\prime \prime}$. The linear addition of power densities $\sum S_{r}^{\prime}$ have the advantage of being slightly faster and less variable (more robust) while the phase correct field strength $\left(\sum E_{r}^{\prime}\right)^{2}$ based computations exhibit a larger dynamic. Power density and field strength can be directly related through free space impedance $Z_{0}$, for the field strength based computation of $\sigma$ :

$$
\begin{aligned}
E_{i} & =\sqrt{S_{i}^{\prime} Z_{0}} \\
E_{r} & =\sum_{m=0}^{N_{r}} E_{r, m}^{\prime} e^{j k d^{\prime}} \\
\sigma & =4 \pi r^{2} \frac{\left|E_{r}\right|^{2}}{\left|E_{i}\right|^{2}} .
\end{aligned}
$$

\section{Implementation issue: real time capable embedding of radar signature generation}

Figure 6 shows the concept level implementation of RASIG. In order to achieve real time capabilities for the developed methods, the independence of rays from each other was exploited. This allows computations to be carried out in parallel which can be done efficiently on GPUs. We used NVIDIA GPUs which come with an framework for raytracing calculations [32]. Even so, the computations for RASIG take up to $100 \mathrm{~ms}$.

To achieve real time capability the state trajectories of objects from the environment simulation are predicted $100 \mathrm{~ms}$ into the future as shown in Fig. 7 part (1). A least squares algorithm is employed for trajectory prediction. The radar signature generation is then performed using the predicted state of objects Fig. 7 part (2). The resulting RS is computed into echo points Fig. 7 part (2) in the same manner as the

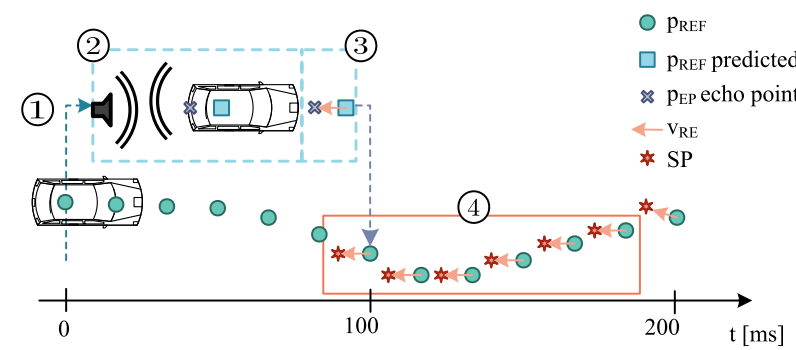

Fig. 7. Real time capable embedding of Radar Signature Generation

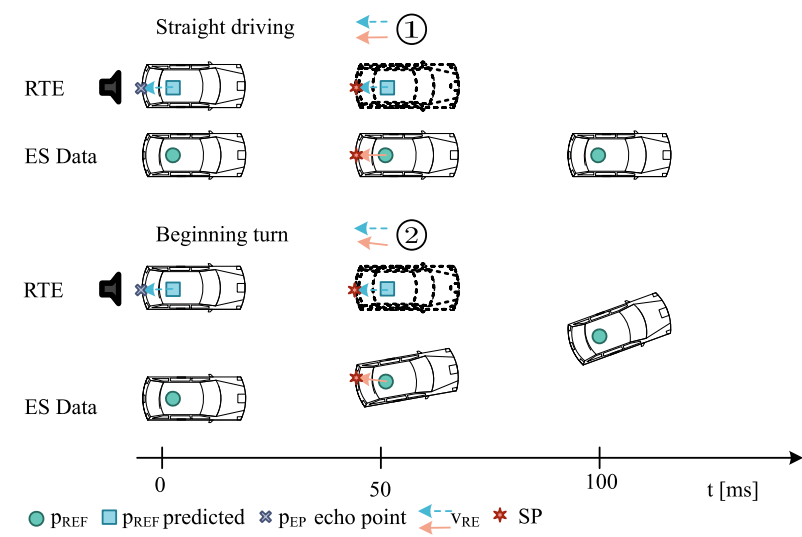

Fig. 8. Accuracy of $V_{R E}$

SP computation described in Sect. 3. The main difference between echo points and SPs is that echo points are computed on a $100 \mathrm{~ms}$ time scale and SPs are computed on a $1 \mathrm{~ms}$ time scale. In order to apply echo points over the next 100 ms time span they are generalized. This is done by computing the vector between the echo point of an object Fig. 7 part 3, typically somewhere on the surface of an object and the predicted reference point of the object using

$$
\mathbf{V}_{R E}=\mathbf{p}_{E P}-\mathbf{p}_{R E F} .
$$

The relation between echo point and reference point $\mathbf{V}_{R E}$ remains fairly constant over $100 \mathrm{~ms}$. For straight driving it is completely constant and thus accurate as shown in Fig. 8 part 11 , with no difference between the real time embedded (RTE) $\mathbf{V}_{R E}$ and a $\mathbf{V}_{R E}$ that would be computed at any time between two RTE computation steps. For turning objects $\mathbf{V}_{R E}$ changes slightly Fig. 8 part (2). As the echo point and $\mathbf{V}_{R E}$ computations start $100 \mathrm{~ms}$ in advance (due to the prediction), their results are available exactly when the predicted time horizon has passed.

A stimulation point is now computed from the current echo point, the REV and the last reference point of the corresponding object from the environment simulation Fig. 7 part (4). The stimulation point contains Doppler shift $f_{D}$ and RCS $\sigma$ from the echo point and the azimuth and distance are computed by adding the computed REV to the reference point. This method allows stimulation points to be updated in less than $1 \mathrm{~ms}$, thus realizing real time capability.

\section{Validation results}

The radar signature simulation and stimulation point RCS computation were validated against more exact field simulations and analytical values. First the RCS was computed for two spheres of different radii $r_{s}$ with RASIG and a commercial field simulation software (CST) with asymptotic far-field solver (i.e. parallel field) for $77 \mathrm{GHz}$. 
Table 1. Sphere results in CST and RASIG

\begin{tabular}{lccl}
\hline$r_{S}[\mathrm{~m}]$ & $r_{S}^{2} \pi\left[\mathrm{dBm}^{2}\right]$ & $\sigma_{C S T}\left[\mathrm{dBm}^{2}\right]$ & $\sigma_{R A S I G_{S_{R}}}\left[\mathrm{dBm}^{2}\right]$ \\
\hline 0.03 & -25.49 & -25.51 & -25.7 \\
0.3 & -5.49 & -5.49 & -5.72 \\
\hline
\end{tabular}

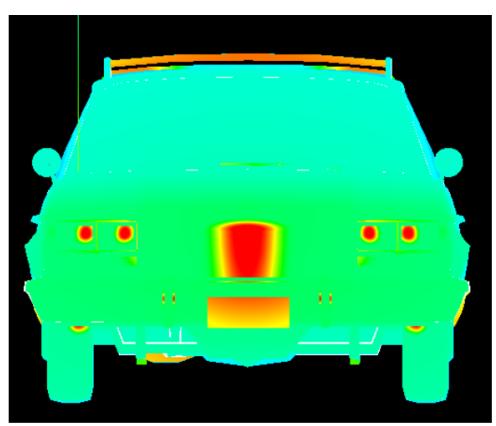

Fig. 9. Phong RS car front

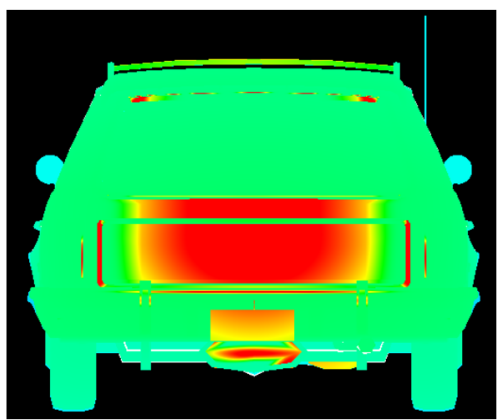

Fig. 10. Phong RS car back

The computations in RASIG were executed with a FoV of: $\phi=17^{\circ}$, $\theta=4.3^{\circ}, \phi_{r}=0.1^{\circ}$ at a distance of $30 \mathrm{~m}$. Material properties were modeled as perfect electric conductors (PEC) and the Phong coefficients were chosen as $k_{s}=1-k_{d}, k_{d}=0.09, n_{s}=700$ (similar to [27]). The results for spheres are given in Table 1 and show good agreement between analytic, CST and RASIG results for varying object sizes.

Second a $360^{\circ}$ monostatic RCS for a car represented as a triangle mesh is computed in CST and in RASIG with Phong-field strength and power density, shown in Fig. 11. The material properties of the car are approximated as perfect electric conductor. Radar signature results are shown in Figs. 9 and 10. One can see that the areas of the car that are perpendicular to the radar sensor reflect more power back than the rest of the car. The computation of monostatic RCS (in steps of $1^{\circ}$ ) for car took over $3 \mathrm{~h}$ in CST, and $36 \mathrm{~s}$ in RASIG. The difference between CST and RASIG especially at the peaks $\left(90^{\circ}, 180^{\circ}\right.$, $270^{\circ}$ in Fig. 11) is in part due to parallel incident field in CST and divergent incident field in RASIG. Also, field strength based computations are sensitive to the distance between rays, which can introduce systematic phase summation errors which explain the local minima at $90^{\circ}$ and $270^{\circ}$ in Fig. 11. The field strength based computations exhibit higher dynamic as stated in Sect. 3.2. But nevertheless, both results show a good agreement in trends.

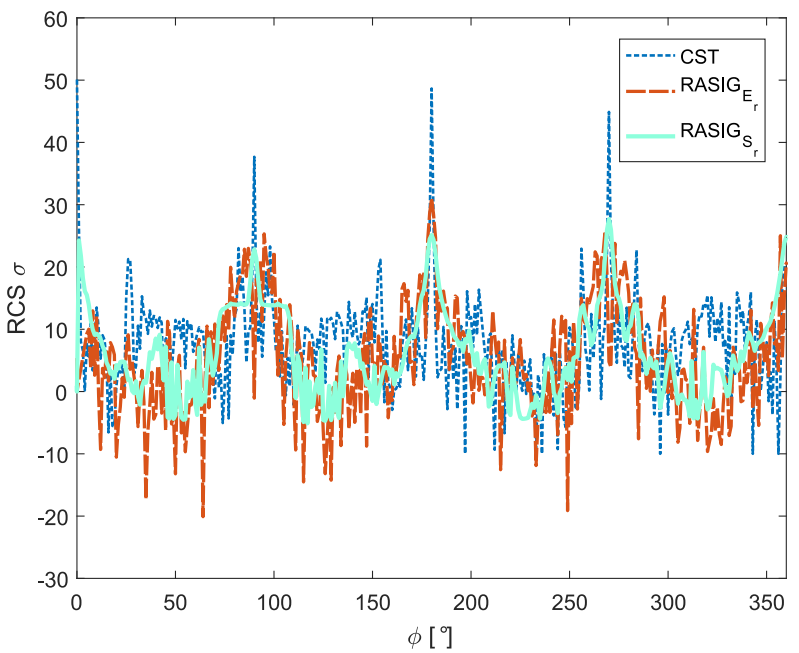

Fig. 11. Comparison of $360^{\circ}$ monostatic car RCS computed in CST vs. RASIG power density and field strength computations

\section{Conclusion}

In this article we presented OTA stimulation chain for automotive radar in ADF ViL testing and discussed requirements on EPSR as well as the OTA stimulation chain. The requirements were derived from the radar sensor as well as the environment simulation side. Current approaches for RCS computation in EPSR were given and the methods employed within RASIG for simulation and stimulation input generation were presented. Details of implementation to realize real-time capability of the stimulation point computation were given and validation results for RASIG were discussed which elucidate both the strengths and weaknesses of our results. In the future multipath RCS and stimulation point computations, parallel field computation for validation as well as validation using real world measurements will be investigated.

\section{Acknowledgements}

Open access funding provided by Graz University of Technology. This work was in part funded by the Austrian Research Promotion Agency (FFG) under the research project GAZELE (No.: 848457).

This work has been partially conducted with in the ENABLE-S3 project which received funding from the ECSEL Joint Undertaking under grant agreement no 692455. This joint undertaking is supported by the European Union's Horizon 2020 Research and Innovation Programme and Austria, Denmark, Germany, Finland, Czech Republic, Italy, Spain, Portugal, Poland, Ireland, Belgium, France, Netherlands, United Kingdom, Slovakia, and Norway.

Open Access This article is distributed under the terms of the Creative Commons Attribution 4.0 International License (http://creativecommons.org/ licenses/by/4.0/), which permits unrestricted use, distribution, and reproduction in any medium, provided you give appropriate credit to the original author(s) and the source, provide a link to the Creative Commons license, and indicate if changes were made.

\section{References}

1. SAE (2016): Taxonomy and definitions for terms related to driving automation systems for on-road motor vehicles.

2. Sovani, S. (2017): Simulation accelerates development of autonomous driving. ATZ Worldw., 119(9), 24-29. https://doi.org/10.1007/s38311-017-0088-y. ISSN 21929076.

3. [Online]. Available: http://www.pegasus-projekt.info/en/.

4. European initiative to enable validation for highly automated safe and secure systems [Online]. Available: https://www.enable-s3.eu. 
5. Riedmaier, S., Nesensohn, J., Gutenkunst, C., Duser, T., Schick, B., Abdellatif, H. (2018): Validation of X-in-the-loop approaches for virtual homologation of automated driving functions. GSVF [Online]. Available: https://www.gsvf.at/program/docs/ fullpapers/04_GSVF2018_Riedmaier_HSKempten.pdf.

6. Schyr, C. (2016): DrivingCube-a novel concept for validation of powertrain and steering systems with automated driving. In Advanced vehicle control: proceedings of the 13th international symposium on Advanced Vehicle Control (AVEC'16).

7. ISPACE: Closed-loop HIL system for testing camera-based systems by inserting data into the image sensor output [Online]. Available: https://www.dspace.com/en/pub/ home/applicationfields/our_solutions_for/driver_assistance_systems/hil_simulation/ closed_loop_hil_testing_camera.cfm.

8. Winner, H., Hakuli, S., Wolf, G. (2012): Handbuch Fahrerassistenzsysteme Grundlagen, Komponenten und Systeme für aktive Sicherheit und Komfort. 2. korrigierte Auflage. ATZ/MTZ-Fachbuch. Wiesbaden: Vieweg/Teubner. ISBN 978-3-8348-1457-9. https://doi.org/10.1007/978-3-8348-8619-4.

9. VIRES Simulationstechnologie GmbH: VTD—Virtual Test Drive [Online]. Available: https://vires.com/vtd-vires-virtual-test-drive.

10. tass international: PreScan: simulation of ADAS \& active safety [Online]. Available: https://www.tassinternational.com/prescan.

11. W.I. GmbH: Radar target simulator $24 \mathrm{GHz}$ and $77 \mathrm{GHz}$ [Online]. Available: http:// www.wks-informatik.de/en/solutions/radar-target-simulator.

12. Radar test bench with dSPACE SCALEXIO HIL system, dSpace [Online]. Available: https://www.dspace.com/en/pub/home/medien/videos/productvideos/video radartestbench.cfm.

13. Keysight Technologies (2016): E8707A radar target simulator $76 \mathrm{GHz}$ to $77 \mathrm{GHz}$ solution brochure, pp. 1-4.

14. Schwarz, S., Rohde, R.: Echo generator for end-of-line production testing of automotive radar sensors, R\&S AREG100A [Online]. Available: https://www.rohde-schwarz. $\mathrm{com} / \mathrm{dk} /$ news-press/press-room/press-releases-detailpages/new-solutions-fromrohde-schwarz-for-automotive-radar-echo-generation-and-radome-measurementsat-eumw-2017-press_releases_detailpage_229356-487812.html?rusprivacypolicy $=0$

15. Gruber, A., Gadringer, M., Schreiber, H., Amschl, D., Bosch, W., Metzner, S., Pflugl, H. (2017): Highly scalable radar target simulator for autonomous driving test beds. In 2017 14th European radar conference (pp. 147-150). Piscataway: IEEE. ISBN 978-2 87487-049-1. https://doi.org/10.23919/EURAD.2017.8249168.

16. Gadringer, M. E., Schreiber, H. (2018): Virtual reality for automotive radars. E\&l, Elektrotech. Inf.tech. https://doi.org/10.1007/s00502-018-0620-9.

17. Gadringer, M. E., Maier, F. M., et al.: Radar target stimulation for automotive applications. IET Radar Sonar \& Navig. J. Accepted, to be published in Oct.

\section{Authors}

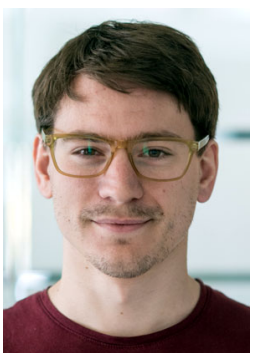

\section{F. Michael Maier}

has been research assistant and Ph.D. student at the Institute of Automation and Control, Graz University of Technology, since 2015. He received the Dipl.-Ing. (M.Sc.) in electrical engineering from the Graz University of Technology in 2014. His main research interests include radar cross section simulation, automated driving and information theory.

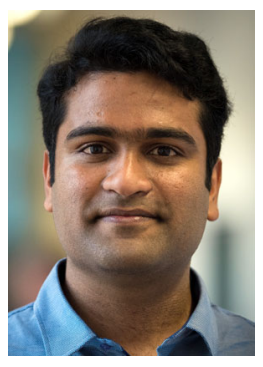

\section{Vamsi P. Makkapati}

received his Ph.D. from the Institute of $\mathrm{Au}$ tomation and Control, Graz University of Technology, Austria. He has eight years of experience in the development and control of autonomous/automated functions for vehicles. His current research is focused on modelling of radar sensors for the purpose of validating various autonomous functions on the present/future vehicles.
18. Elking, D. M., Roedder, J. M., Car, D. D., Alspach, S. D. (1995): A review of highfrequency radar cross section analysis capabilities at McDonnell Douglas Aerospace. IEEE Antennas Propag. Mag., 37(5), 33-42. https://doi.org/10.1109/74.475862. ISSN 10459243.

19. Taflove, A., Umashankar, K. R. (1989): Review of FD-TD numerical modeling of electromagnetic wave scattering and radar cross section. Proc. IEEE, 77(5), 682-699. https://doi.org/10.1109/5.32059. ISSN 00189219.

20. Bel Kamel, E., Peden, A., Pajusco, P. (2017): RCS modeling and measurements for automotive radar applications in the W band. In 2017 11th European Conference on Antennas and Propagation (EUCAP) (pp. 2445-2449). Piscataway: IEEE. ISBN 978-88907-0187-0. https://doi.org/10.23919/EuCAP.2017.7928266.

21. Bernsteiner, S., Magosi, Z., Lindvai-Soos, D., Eichberger, A. (2015): Radar sensor model for the virtual development process. ATZ-Elektron. Worldw., 10(2), 46-52. ISSN 2192-9092.

22. Hirsenkorn, N., Hanke, T., Rauch, A., Dehlink, B., Rasshofer, R., Biebl, E. (2016): Virtual sensor models for real-time applications. Adv. Radio Sci., 14(B), 31-37. ISSN 16849965.

23. Schuler, K., Becker, D., Wiesbeck, W. (2008): Extraction of virtual scattering centers of vehicles by ray-tracing simulations. IEEE Trans. Antennas Propag., 56(11), 3543-3551. https://doi.org/10.1109/TAP.2008.2005436.

24. Maxwell, J. C., Torrance, T. F. (1996): A dynamical theory of the electromagnetic field. The Torrance collection. Eugene: Wipf and Stock. [Online]. Available: https://books. google.at/books?id=el4pAQAAMAAJ. ISBN 9781579100155.

25. Pharr, M., Jakob, W., Humphreys, G. (2016): Physically based rendering: from theory to implementation. San Francisco: Morgan Kaufmann.

26. Phong, B. T. (1975): Illumination for computer generated pictures. Commun. ACM, 18(6), 311-317. ISSN 00010782

27. Maier, F. M., Makkapati, V. M., Horn, M. (2018): Adapting Phong into a simulation for stimulation of automotive radar sensors. In IEEE international conference on microwaves for intelligent mobility.

28. Balanis, C. A. (2012): Advanced engineering electromagnetics. CourseSmart series. New York: Wiley.

29. Purcell, E. (2011): Electricity and magnetism. Cambridge: Cambridge University Press. ISBN 9781139503556

30. Buck, J. A., Hayt, W. H. (2011): Engineering electromagnetics. New York: McGraw-Hill Education. ISBN 9780073380667.

31. Stratton, J. A., Antennas, I., Society, P. (2007): Electromagnetic theory An IEEE Press classic reissue. New York: Wiley. [Online]. Available: https://books.google.at/ books?id=zFeWdS2luE4C. ISBN 9780470131534.

32. NVIDIA: NVIDIA® OptiXTM ray tracing engine [Online]. Available: https://developer. nvidia.com/optix.

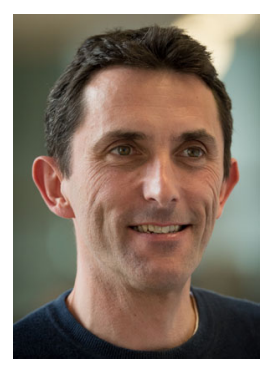

\section{Martin Horn}

is head of the Institute of Automation and Control at Graz University of Technology, Austria. In 2003 he became associate professor at TU Graz, in 2008 he was appointed as a full professor for control and measurement systems at Klagenfurt University, Austria. In 2014 he became full professor at TU Graz. He has been leader of various research projects and is author of numerous publications. His research interests include nonlinear robust control, especially variable structure systems, modelling, simulation and control of mechatronic systems. 